\title{
Pulmonary hypertension complicating pulmonary sarcoidosis
}

\author{
M. P. Huitema ${ }^{1}$ J. C. Grutters ${ }^{2,3}$ • B. J. W. M. Rensing ${ }^{1}$ H. J. Reesink ${ }^{2}$ M. C. Post ${ }^{1}$
}

Published online: 18 May 2016

(C) The Author(s) 2016. This article is available at SpringerLink with Open Access

\begin{abstract}
Pulmonary hypertension (PH) is a severe complication of sarcoidosis, with an unknown prevalence. The aetiology is multifactorial, and the exact mechanism of $\mathrm{PH}$ in the individual patient is often difficult to establish. The diagnostic work-up and treatment of $\mathrm{PH}$ in sarcoidosis is complex, and should therefore be determined by a multidisciplinary expert team in a specialised centre. It is still a major challenge to identify sarcoidosis patients at risk for developing PH. There is no validated algorithm when to refer a patient suspected for $\mathrm{PH}$, and $\mathrm{PH}$ analysis itself is difficult. Until present, there is no established therapy for PH in sarcoidosis. Besides optimal treatment for sarcoidosis, case series evaluating new therapeutic options involving PH-targeted therapy are arising for a subgroup of patients. This review summarises the current knowledge regarding the aetiology, diagnosis and possible treatment options for $\mathrm{PH}$ in sarcoidosis.
\end{abstract}

Keywords Pulmonary hypertension - Sarcoidosis . Interstitial lung disease

M. P. Huitema

mp.huitema@antoniusziekenhuis.nl

1 Department of Cardiology, St. Antonius Hospital Nieuwegein, Nieuwegein, The Netherlands

2 Department of Pulmonology, St. Antonius Hospital Nieuwegein, Nieuwegein, The Netherlands

3 Division of Heart and Lung, UMC Utrecht, Utrecht, The Netherlands

\section{Introduction}

Sarcoidosis is a rare, multisystemic disorder of unknown aetiology. It is characterised by non-caseating granulomas which present in multiple tissues, particularly in the lung and lymphatic system. The course of sarcoidosis can vary from spontaneous resolution to severe and chronic disease. It is often complicated by pulmonary fibrosis [1].

Pulmonary hypertension $(\mathrm{PH})$ is defined as a mean pulmonary artery pressure (PAP) of $\geq 25 \mathrm{mmHg}$ at rest measured by right heart catheterisation [2]. $\mathrm{PH}$ is increasingly recognised as a serious complication of pulmonary sarcoidosis. The clinical diagnosis or suspicion of $\mathrm{PH}$ in sarcoidosis is challenging, since symptoms overlap. $\mathrm{PH}$ exists in all stages of sarcoidosis; however, the frequency largely depends on the severity of the sarcoidosis [3, 4]. There is a lack of robust data on accurate diagnostic tools and therapeutic options for $\mathrm{PH}$ in sarcoidosis. However, diagnostic tests are in further development, and case series of potential treatment options are arising. Diagnosis and treatment decisions should be made in a specialised team, including a dedicated cardiologist, pulmonologist, radiologist and nurse. In this review, we will discuss the classification and aetiology of sarcoidosis-associated PH (SAPH), diagnostic tests and possible treatment options.

\section{Epidemiology}

There are no clear data regarding the true prevalence of SAPH. Rates of PH in sarcoidosis patients in tertiary centres vary between $5-20 \%[3,5,6]$. As a side note, the gold standard right heart catheterisation for diagnosing $\mathrm{PH}$ is often absent in studies, where results are based on echocardiography only. The prevalence of PH may increase to 
Tab. 1 Clinical classification of pulmonary hypertension [2]

\begin{tabular}{|c|c|}
\hline WHO group 1 & Pulmonary arterial hypertension \\
\hline WHO group 2 & Pulmonary hypertension due to left heart disease \\
\hline WHO group 3 & Pulmonary hypertension due to lung disease and/or hypoxaemia \\
\hline WHO group 4 & Chronic thromboembolic pulmonary hypertension or other pulmonary artery obstructions \\
\hline \multirow[t]{5}{*}{ WHO group 5} & Pulmonary hypertension with unclear multifactorial mechanism \\
\hline & 5.1 Hematologic disorders: myeloproliferative disorders, splenectomy, chronic haemolytic disorders \\
\hline & Systemic disorders: sarcoidosis, pulmonary Langerhans cell histiocytosis lymphangioleiomyomatisos \\
\hline & Metabolic disorders: glycogen storage disease, Gaucher disease, thyroid disorders \\
\hline & $\begin{array}{l}\text { Others: pulmonary tumoral thrombotic microangiopathy, fibrosing mediastinitis, chronic renal failure, } \\
\text { segmental pulmonary hypertension }\end{array}$ \\
\hline
\end{tabular}

WHO World Health Organisation

$>50 \%$ in sarcoidosis patients with persistent or unexplained dyspnoea [4, 7]. A PH prevalence of more than $70 \%$ was found in sarcoidosis patients awaiting lung transplant $[8,9]$.

\section{Prognosis}

Mortality in sarcoidosis patients is significantly increased if $\mathrm{PH}$ is present, independent of pulmonary function [10, 11]. For 22 patients with haemodynamically confirmed SAPH, the 1-, 2-, and 5-year survival was found to be 84 , 74 and $59 \%$, compared with 100, 96 and $96 \%$ in matched sarcoidosis patients without $\mathrm{PH}(p=0.003)$ [10]. Another, non-invasive study showed an echocardiographic estimation of systolic PAP $>50 \mathrm{mmHg}$ to be associated with increased mortality [7].

\section{Classification of PH in sarcoidosis}

According to the World Health Organisation, PH is classified into five different groups, based on clinical presentation, pathological findings, haemodynamic characteristics and treatment strategy (Tab. 1; [2]). SAPH is classified in group 5: $\mathrm{PH}$ with an unknown and multifactorial mechanism. However, PH in sarcoidosis can be classified in group 2, 3 or 4 as well, if the underlying mechanism is clear. For example, a patient with severe fibrotic abnormalities with a mean PAP that is in line with the severity of the lung disease will be classified as group $3 \mathrm{PH}$. On the other hand, if a patient presents with mild lung disease, but the mean PAP is high (e.g. $>35 \mathrm{mmHg}$ ), a component of vasculopathy can be suspected. The haemodynamic profile might show similarities with pulmonary arterial hypertension. Because of the unknown mechanism, this patient will be classified as group $5 \mathrm{PH}$.

\section{Aetiology of PH in sarcoidosis}

The aetiology of PH in sarcoidosis is multifactorial. Based on the pulmonary artery wedge pressure (PAWP), SAPH can be divided into pre-capillary (PAWP $\leq 15 \mathrm{mmHg}$ ) and post-capillary (PAWP $>15 \mathrm{mmHg}$ ) $\mathrm{PH}[2]$.

\section{Pre-capillary PH}

Pre-capillary PH in sarcoidosis might be due to one or several mechanisms. Hypothesised mechanisms are described below.

\section{Destruction of distal capillary bed and resultant} hypoxaemia

Hypoxic vasoconstriction due to destruction of the distal capillary bed is one of the first possible mechanisms of SAPH. Data to support this mechanism are scarce, ranging from no difference in oxygen saturation or need for supplemental oxygen between sarcoidosis patients with and without PH [4], to the need of supplemental oxygen as only predictor of SAPH in patients listed for lung transplantation [8]. The majority of patients with SAPH suffer a more advanced stage of sarcoidosis, often in the presence of pulmonary fibrosis $[3,5,7,10]$. However, approximately $32-50 \%$ of the patients with SAPH have no significant fibrosis $[3,5,10]$, suggesting that fibrotic ablation of the pulmonary vasculature is not the only mechanism to explain SAPH.

\section{Specific vasculopathy}

Involvement of granulomatous vasculopathy is another possible mechanism of SAPH [12]. One study reported vascular involvement in all 40 autopsies of patients with mainly pulmonary and cardiac sarcoidosis [13]. Granulomatous vasculopathy causes frequent occlusion of arterioles or venules [13] and angiitis [14], which might result in PH. 
Fig. 1 Compression of the pulmonary artery. A case of a 60-year-old female with stage IV sarcoidosis, diagnosed with $\mathrm{PH}$ with a mean PAP of $63 \mathrm{mmHg}$, due to compression of the pulmonary artery. a Compression on chest $\mathrm{CT}$; b Pulmonary angiogram of the left lobe
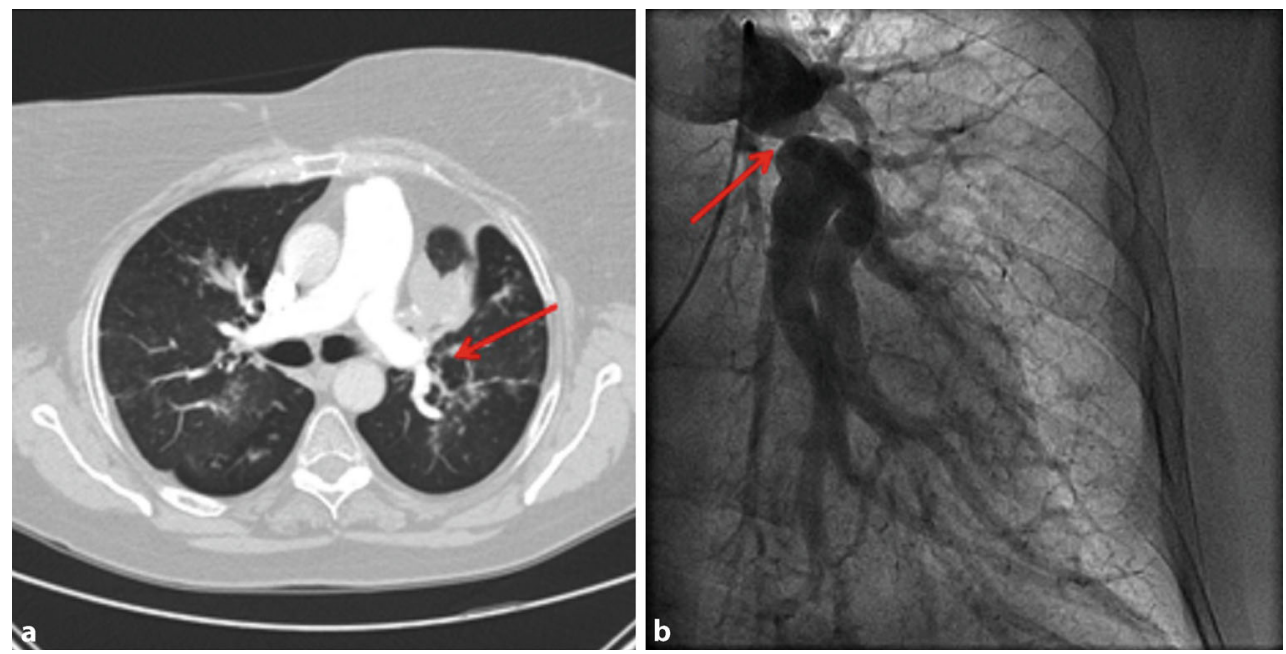

Sarcoid granulomas can be found throughout all layers of the pulmonary vascular wall, often in combination with intimal fibrosis, medial proliferation, inflammatory changes, necrosis and destruction of the small-vessel architecture, which leads to an occlusive vasculopathy [10,13]. Granulomas can also be formed at the level of the intima in large vessels. These patients are often misclassified as having chronic thromboembolic pulmonary hypertension.

\section{Local increased vasoreactivity}

Acute vasoresponsiveness is defined as a decrease in mean PAP of $\geq 10 \mathrm{mmHg}$ to an absolute value of mean PAP $\leq 40 \mathrm{mmHg}$, with an increased or unchanged cardiac output [2]. Several patients with SAPH have shown responsiveness to acute vasodilator challenge with inhaled nitrogen oxide or prostacyclin. Preston et al. [15] showed a decrease of $18 \pm 4 \%$ in mean PAP and $31 \pm 5 \%$ in pulmonary vascular resistance in seven of eight patients receiving inhaled nitric oxide, with a slight increase of cardiac output. For epoprostenol, no significant change was reported. Fisher et al. [16] showed an average decrease in pulmonary vascular resistance of $45 \%$, and a non-significant decrease in mean PAP of $11 \%$ in six out of seven patients using epoprostenol. However, an increased vasoreactivity is not a predictor for response to PH-targeted therapy in pulmonary arterial hypertension, but highlights the potential role of an increased reactivity of the pulmonary vasculature as part of the unclear pathogenesis of $\mathrm{PH}$ in sarcoidosis.

\section{Extrinsic compression of pulmonary vessels}

Extrinsic compression of the pulmonary arteries, and less frequently the pulmonary veins, by enlarged lymph nodes or fibrosing mediastinitis, has also been described as a mech- anism for SAPH [10, 13, 17]. Fig. 1 shows an example of a sarcoidosis patient with PH due to compression of the pulmonary artery. Compression of the pulmonary artery occurs more frequently in chronic sarcoidosis, and presented in $21 \%$ of the PH patients with radiographic stage IV sarcoidosis [10].

\section{Portal hypertension}

Hepatic sarcoidosis is present in up to $70 \%$ of sarcoidosis patients, and might lead to $\mathrm{PH}$ due to cirrhosis and portal hypertension [18]. Approximately $1-5 \%$ of patients with portal hypertension develop PH [2], with liver cirrhosis being the most common cause of portal hypertension.

\section{Pulmonary veno-occlusive disease}

Pulmonary veno-occlusive disease is defined as extensive narrowing or occlusion of the pulmonary veins in lobular septa or intra-acinar venules by fibrous tissue [19]. In a case series of five patients with SAPH who underwent lung transplant, all explanted lungs demonstrated presence of pulmonary veno-occlusive disease [10].

\section{Post-capillary PH}

Apart from pre-capillary $\mathrm{PH}$, post-capillary $\mathrm{PH}$ can also be found in sarcoidosis. Systolic, diastolic and subclinical left ventricular dysfunction are often present in sarcoidosis [20]. In a retrospective study consisting of 130 sarcoidosis patients of all stages, with persistent moderate to severe dyspnoea, who underwent right heart catheterisation after at least six months of immunosuppressive therapy, the prevalence of $\mathrm{PH}$ was $54 \%$. Interestingly, in $29 \%$ an elevated PAWP $>15 \mathrm{mmHg}$ was measured, suggesting left ventricular disease as a cause of PH [7]. Left ventricular 
Fig. 2 Measurement of the pulmonary artery on chest $\mathrm{CT}$. a Measurement of the main pulmonary artery, at the level of the bifurcation along the line that originates from the centre of the adjacent ascending aorta, perpendicular to the axis of main pulmonary artery; $\mathbf{b}$ If the trunk is too curved, measurements can be made using the other method [32]
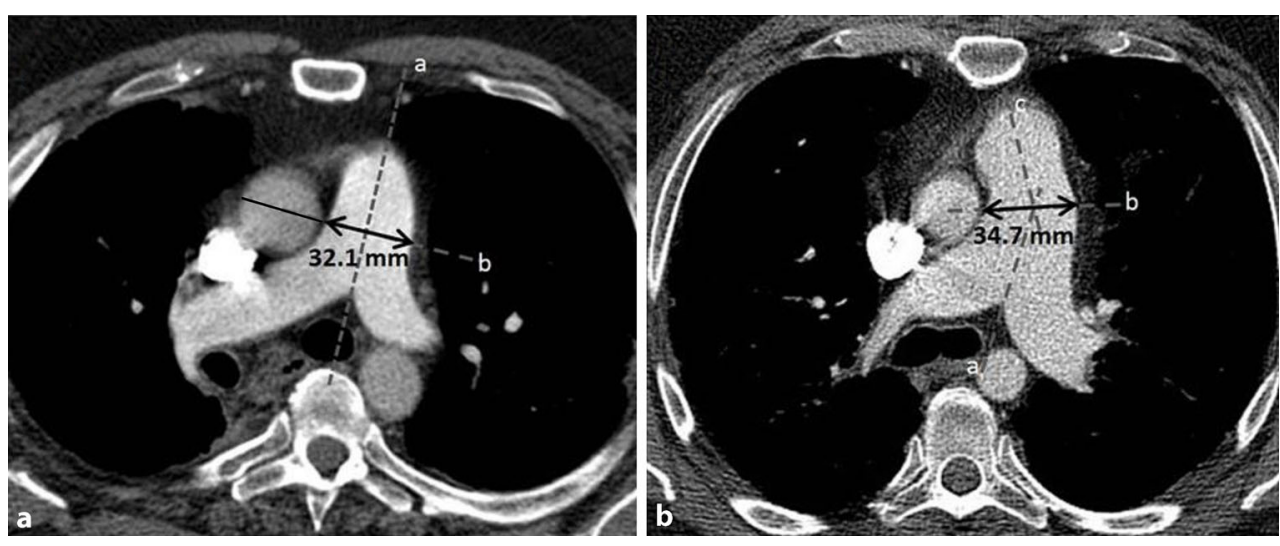

dysfunction might be due to myocardial involvement of sarcoidosis. Autopsy studies suggest that at least $25 \%$ of the patients with sarcoidosis have myocardial involvement. The diagnosis of cardiac sarcoidosis is difficult, because signs and symptoms can be subtle or even absent. The expert consensus statement advises to screen for cardiac sarcoidosis using cardiac magnetic resonance imaging (CMR) and 18 F-fluorodeoxyglucose positron emission tomography in sarcoidosis patients at risk for myocardial involvement [21].

\section{Diagnosis}

Establishing the diagnosis of $\mathrm{PH}$ in sarcoidosis is challenging. Symptoms suggestive for PH are dyspnoea, dizziness, cough and chest pain, or signs such as elevated jugular venous pressure, S3 or S4 heart sounds, lower extremity oedema or right ventricular heave. These signs are usually associated with more advanced PH [4]. During followup, several tests may raise suspicion of $\mathrm{PH}$, requiring $\mathrm{PH}$ analysis.

\section{Suspicion of PH}

\section{Pulmonary function test}

Several parameters of pulmonary function testing are associated with SAPH, such as a decreased forced vital capacity, total lung capacity [5] and decreased diffusion capacity of carbon monoxide [3]. However, PH also occurs in sarcoidosis patients with near-normal lung function tests. An echocardiographic study reports a prevalence of $\mathrm{PH}$ of $28 \%$ in 127 sarcoidosis patients with near-normal lung function tests, defined as a forced vital capacity $>70 \%$, forced expiratory volume in 1 second $>70 \%$, and diffusion capacity of carbon monoxide $>60 \%$ [22]. Therefore, $\mathrm{PH}$ is suspected in the presence of progressive dyspnoea, while pulmonary function tests remain stable.

\section{Exercise testing}

The 6-minute walk test (6MWT) can also raise suspicion for PH. Most sarcoidosis patients with PH will have a 6MWT distance of less than 450 meters [3, 23-27]. An oxygen desaturation to a value below $90 \%$ during the 6MWT is associated with PH [3]. However, a major limitation of the 6MWT is that results may be influenced by other factors, such as airway disease, cardiac disease, fatigue and muscle involvement [27].

Cardiopulmonary exercise testing for detecting $\mathrm{PH}$ has not been studied systematically in sarcoidosis. In patients with pulmonary arterial hypertension, reduced maximum oxygen uptake (VO2max), increased ventilator inefficiency, early lactate acidosis, decreased end-expiratory $\mathrm{CO} 2$ and reduced $\mathrm{O} 2$ pulse are an indication for pulmonary arterial hypertension, although these parameters are non-specific [28]. One study evaluated exercise testing in patients with interstitial lung disease. Peak exercise capacity was significantly lower in interstitial lung disease patients with PH compared with those without PH. Also, carbon dioxide production and load (Watts) was lower, and the ventilation/ carbon dioxide production ratio at ventilatory threshold was higher in patients with $\mathrm{PH}$ [29].

\section{Chest computed tomography}

A dilated pulmonary artery on chest computed tomography (CT) may raise suspicion of PH [2]. The cut-off value is $\geq 29 \mathrm{~mm}$ for the pulmonary artery, or a ratio of the pulmonary artery diameter to the ascending aorta diameter of $\geq 1$ [2]. Fig. 2 shows the measurement of the pumonary artery diameter on chest CT. Two studies $[30,31]$ found no correlation between the pulmonary artery diameter and the mean PAP in patients with fibrotic lung disease. However, a recently published article investigating only sarcoidosis patients [32], showed a good correlation for the pulmonary artery diameter indexed for body surface area and the presence of PH; this had a high diagnostic accuracy for discrimi- 
Tab. 2 Echocardiographic probability of pulmonary hypertension in symptomatic patients with a suspicion of $\mathrm{PH}$ [2]

\begin{tabular}{|c|c|c|}
\hline $\begin{array}{l}\text { Echocardiographic pulmonary hyperten- } \\
\text { sion probability }\end{array}$ & $\begin{array}{l}\text { Peak tricuspid regurgitation velocity } \\
(\mathrm{m} / \mathrm{s})\end{array}$ & $\begin{array}{l}\text { Other signs }{ }^{\mathrm{a}} \text { for pulmonary hypertension on } \\
\text { echocardiography }\end{array}$ \\
\hline Low & $\leq 2.8$ or not measurable & No \\
\hline \multirow[t]{2}{*}{ Intermediate } & $\leq 2.8$ or not measurable & Yes \\
\hline & $2.9-3.4$ & No \\
\hline \multirow[t]{2}{*}{ High } & $2.9-3.4$ & Yes \\
\hline & $>3.4$ & Not required \\
\hline
\end{tabular}

${ }^{a}$ Other signs are described in Tab. 3. To meet the criteria of other signs, at least two other signs of at least two different categories must be abnormal

Tab. 3 Echocardiographic signs suggestive for pulmonary hypertension [2]

The ventricles Right ventricle/left ventricle basal diame-
ter ratio $>1.0$

Pulmonary artery

Inferior vena cava and right atrium
Pulmonary arterial acceleration time $<105$ msec and/or midsystolic notching

Inferior cava diameter $>21 \mathrm{~mm}$ with decreased inspiratory collapse $(<50 \%$ with a sniff or $<20 \%$ with quiet inspiration)
Flattening of the interventricular septum

(left ventricular eccentricity index $>1.1$ in systole and/or diastole)

Early diastolic pulmonary regurgitation velocity $>2.2 \mathrm{~m} / \mathrm{sec}$

PA diameter

Right atrial area (end-systole $>18 \mathrm{~cm}^{2}$ )

$P A$ pulmonary artery, $P H$ pulmonary hypertension

nating between the presence and absence of PH. Other chest CT parameters are right ventricular enlargement, or a segmental artery:bronchus ratio $>1: 1$. However, they seem to have less value in clinical practice.

\section{Cardiac magnetic resonance imaging}

CMR is often used for detecting cardiac sarcoidosis. In case of suspicion of PH, CMR is a valuable tool to accurately and reproducibly assess the right ventricular dimension, morphology and function, stroke volume, cardiac output, pulmonary artery distensibility and right ventricular mass. Reduced pulmonary artery distensibility and retrograde flow are predictive for $\mathrm{PH}$ in patients at risk; however, this cannot exclude $\mathrm{PH}$ [33].

\section{Specific PH analysis}

In general, the $\mathrm{PH}$ analysis protocol used in sarcoidosis patients is similar to the standard analysis protocol as described in the $\mathrm{PH}$ guideline [2]. The analysis consists of biomarkers, electrocardiogram (ECG) and echocardiography.

\section{Biomarkers}

The main biomarker for $\mathrm{PH}$ is brain natriuretic peptides (BNP). Recently, BNP has been studied in a right heart catheterisation study of a group of 113 patients with interstitial lung disease [34]. BNP was significantly increased in patients with an invasive systolic PAP $>40 \mathrm{mmHg}$. How- ever, BNP levels were significantly higher in patients with an increased PAWP. The correlation between BNP and the mean PAP was strong $(\mathrm{r}=0.82, p<0.001)$.

\section{Electrocardiogram}

Abnormalities on the ECG suggesting right ventricular overload often present in more severe PH. Therefore, ECG cannot be used to exclude the diagnosis. ECG abnormalities include P-pulmonale, right axis deviation, right ventricular hypertrophy or strain, right bundle branch block and QTc prolongation [2].

\section{Echocardiography}

Echocardiography plays a key role in $\mathrm{PH}$ analysis [2]. The primary echocardiographic parameter for $\mathrm{PH}$ is the estimation of the systolic PAP based on the peak tricuspid regurgitation velocity (TRV) measured by continuous wave Doppler and the right atrial pressure [2]. Importantly, this measurement can overestimate or underestimate the actual pressures. A meta-analysis of 29 studies showed a modest diagnostic accuracy [35]. However, the accuracy drops if the tricuspid regurgitation jet is difficult to obtain, and if image quality is limited. Therefore, isolated use of the peak TRV is unreliable. Recently, the guidelines recommend using peak TRV in combination with additional parameters in order to divide patients according to the echocardiographic probability of $\mathrm{PH}$ [2]. Additional parameters include for example right ventricular dilatation and/or hypertrophy, decreased systolic right ventricular function by assessing tri- 
Fig. 3 Flow chart for pulmonary hypertension screening in sarcoidosis

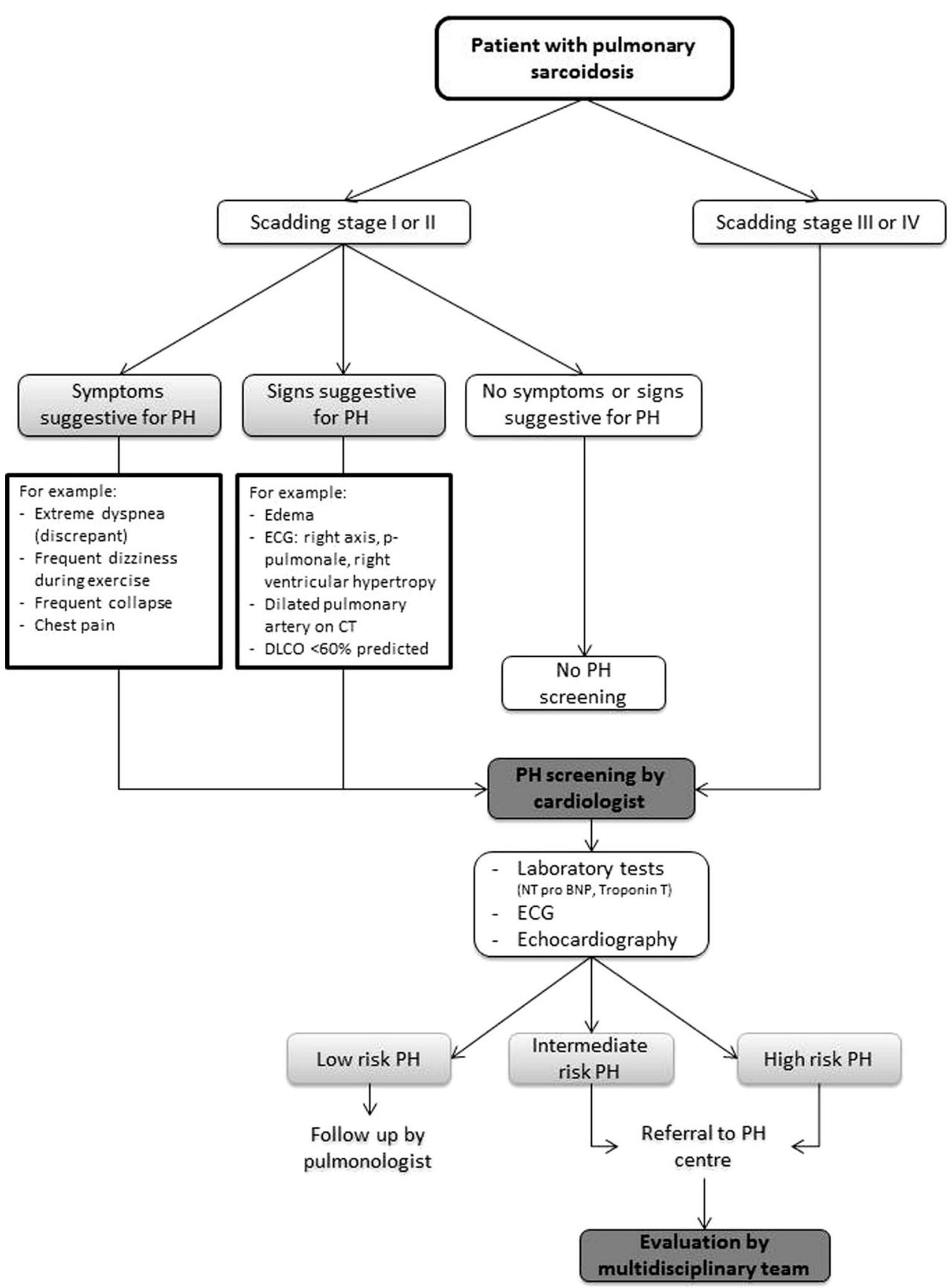

cuspid annulus plane systolic excursion and S-wave on tissue Doppler imaging, pulmonary artery dilation, right atrial dilation, and pulmonary artery acceleration time [2]. The echocardiographic signs and subsequent classifications are summarised in Tab. 2 and 3.

The literature on echocardiographic findings in sarcoidosis patients is scarce, and the value of right ventricular systolic pressure and peak TRV is controversial. In patients with interstitial lung disease including pulmonary fibrosis, right ventricular systolic pressure was found to be less accurate and measurable in only $54 \%$ of the patients [36, 37]. In an echocardiographic study of 80 sarcoidosis pa- tients, TRV was measurable in $70 \%$ of patients. There was a significant correlation $(\mathrm{r}=0.62, p<0.0001)$ between the non-invasive and invasive measurements. However, several patients could have been misclassified [7].

A benefit of echocardiography is the possibility to detect other cardiac abnormalities to explain dyspnoea. Besides $\mathrm{PH}$, the right ventricular function is also associated with cardiac sarcoidosis and abnormal pulmonary function tests, and might even be an isolated finding [38]. 
Tab. 4 Overview of literature on pulmonary hypertension targeted treatment in sarcoidosis

\begin{tabular}{|c|c|c|c|c|c|c|c|}
\hline Author & $\begin{array}{l}\text { Year and } \\
\text { type }\end{array}$ & Treatment & $N$ & mPAP & PVR & $\mathrm{CO}$ & $\begin{array}{l}\text { Other outcomes and adverse } \\
\text { events }\end{array}$ \\
\hline \multicolumn{8}{|c|}{ Prostacyclin analogues } \\
\hline Preston [15] & $2001 \mathrm{CS}$ & Nitric oxide & 8 & $\downarrow 18 \%$ & $\downarrow 31 \%$ & $\uparrow 12 \%$ & \\
\hline Preston [15] & $2001 \mathrm{CS}$ & Epoprostenol & 6 & $\leftrightarrow$ & $\downarrow 25 \%$ & $\uparrow 25 \%$ & \\
\hline Fisher [16] & $2006 \mathrm{CS}$ & Epoprostenol & 7 & $\downarrow 21 \%$ & $\downarrow 45 \%$ & $\uparrow 44 \%$ & $\begin{array}{l}\uparrow \text { NYHA } 1-2 \text { classes } \\
\text { Decreased Pao } 2 \text { in } 3 / 7 \\
\text { One death }\end{array}$ \\
\hline Baughman [45] & $2009 \mathrm{CS}$ & Iloprost & 15 & $\downarrow 15 \%$ & $\downarrow 45 \%$ & - & $\begin{array}{l}\uparrow 12 \% \text { 6MWT } \\
\uparrow \text { QOL } \\
\text { Decrease in Pao2 in 2/15 }\end{array}$ \\
\hline \multicolumn{8}{|c|}{ Endothelin receptor antagonists } \\
\hline Judson [46] & $2011 \mathrm{CS}$ & Ambrisentan & 21 & - & - & - & $\begin{array}{l}\leftrightarrow \text { 6MWT, NYHA, QOL } \\
38 \% \text { discontinued due to } \\
\uparrow \text { oedema and dyspnoea }\end{array}$ \\
\hline Baughman [26] & $2014 \mathrm{RCT}$ & Bosentan & 35 & $\downarrow 11 \%$ & $\downarrow 28 \%$ & - & $\leftrightarrow 6 \mathrm{MWT}$ \\
\hline \multicolumn{8}{|c|}{ Phosphodiesterase 5 inhibitors } \\
\hline Milman [9] & $2008 \mathrm{CS}$ & Sildenafil & 12 & $\downarrow 19 \%$ & $\downarrow 48 \%$ & $\uparrow 36 \%$ & $\leftrightarrow 6 \mathrm{MWT}$ \\
\hline \multicolumn{8}{|l|}{ Combined studies } \\
\hline Barnett [25] & $2009 \mathrm{CS}$ & $\begin{array}{l}\text { Sildenafil, bosen- } \\
\text { tan, epoprostenol }\end{array}$ & 22 & $\downarrow 20 \%$ & $\downarrow 39 \%$ & $\leftrightarrow$ & $\uparrow 6 \mathrm{MWT}$ \\
\hline Dobarro [47] & 2013 CS & $\begin{array}{l}\text { Bosentan (2) or } \\
\text { sildenafil (9) }\end{array}$ & 11 & $\leftrightarrow$ & $\leftrightarrow$ & $\leftrightarrow$ & $\begin{array}{l}\uparrow 6 \mathrm{MWT} \\
\leftrightarrow \text { NYHA }\end{array}$ \\
\hline
\end{tabular}

6MWT six-minute walk test, $C O$ cardiac output, $C S$ case series, $m P A P$ mean pulmonary artery pressure, NYHA New York Heart Association, $\mathrm{PaO} 2$ partial arterial oxygen pressure, $P V R$ pulmonary vascular resistance, $Q o L$ quality of life, $R C T$ randomised controlled trial

\section{Right heart catheterisation}

Right heart catheterisation remains the gold standard for diagnosing $\mathrm{PH}$ [2]. The invasive nature of this diagnostic modality makes it unsuitable for routine use [39]. Right heart catheterisation is recommended in patients with an intermediate or high risk of $\mathrm{PH}$, on echocardiography, with realistic treatment possibilities [2]. The mean PAP in patients awaiting lung transplantation was $9 \mathrm{mmHg}$ higher in sarcoidosis patients compared with idiopathic pulmonary fibrosis, despite similar spirometric severity [40]. In patients with sarcoidosis, PAPs are often higher than expected by parenchymal involvement only [40]. In such cases, a mean PAP exceeding $35 \mathrm{mmHg}$ has to be considered to be severe $\mathrm{PH}$. Additional to interstitial lung disease, these patients are suspected for pulmonary vascular abnormalities [41].

\section{Recommendations for clinical practice}

The literature regarding PH in sarcoidosis is scarce. Therefore, it is difficult to make evidence-based and clear recommendations as to which patients are at risk of developing $\mathrm{PH}$, and on the best method for screening. Based on the current literature, we constructed a flow chart to give some guidance for screening (Fig. 3). Importantly, patients with intermediate to high risk for $\mathrm{PH}$ should be referred to a $\mathrm{PH}$ centre for further analysis.

\section{Management}

In PH, a multidisciplinary approach involving cardiologists, pulmonologists and radiologists specialised in $\mathrm{PH}$ and interstitial lung disease is mandatory. Treatment of PH in sarcoidosis has only been studied in small groups, and there is no solid proof for the use of PH-targeted therapy in sarcoidosis. Therefore, treatment might benefit the individual patient, but there is no evidence for effectiveness. The treatment goal is to improve the vascular, haemodynamic and functional outcomes. Suggested therapies are targeted to the underlying mechanisms of $\mathrm{PH}$ in sarcoidosis. These strategies can be divided into sarcoidosis-targeted treatment and $\mathrm{PH}$-targeted treatment. Both strategies will be described below.

\section{Sarcoidosis-targeted treatment}

Sarcoidosis-targeted treatment might be indicated if the mechanism of PH is suspected to be due to sarcoidosis itself, for example in patients with compression of the pulmonary artery by lymphadenopathy. There is a step-wise approach for the management of sarcoidosis [42]. First- 
line treatment of sarcoidosis consists of oral glucocorticoids. However, long-term use on high doses is associated with substantial morbidity. As an alternative, antimetabolites such as methotrexate and azathioprine might be used. The next step would be anti-tumour necrosis factor monoclonal antibodies, or rituximab [42].

\section{Anti-inflammatory and immunomodulatory agents}

Anti-inflammatory and immunomodulatory agents potentially improve $\mathrm{PH}$, if caused by active granulomatous inflammation. Nunes et al. [10] evaluated ten sarcoidosis patients (one with stage 0, four with stage II, and five with stage IV) after treatment with high doses of glucocorticosteroids. Patients with stage IV sarcoidosis did not respond, whereas in three patients (one with stage 0 and two with stage II) systolic PAP decreased by more than $20 \%$ after 3-6 months of follow-up. Afterwards, these patients were maintained on low doses of corticosteroids, and the systolic PAP continued to decrease. This suggests that fibrotic disease is most likely not responsive to corticosteroids, whereas patients with active granulomatous inflammation might benefit. Patients with compression of the pulmonary artery due to enlarged lymph nodes might also benefit from immunosuppressive treatment [10].

\section{Oxygen therapy}

Hypoxic vasoconstriction due to decreased arterial oxygen pressure is one of the possible mechanisms for $\mathrm{PH}$. Oxygen therapy is recommended in a subgroup of patients. Oxygen administration was shown to reduce pulmonary vascular resistance in patients with pulmonary arterial hypertension; however, there are no randomised data for long-term oxygen therapy [2]. Based on a study in patients with chronic obstructive pulmonary disease, the European guidelines for $\mathrm{PH}$ recommend the use of oxygen in patients with a partial arterial blood oxygen pressure $<8.0 \mathrm{kPa}$ [2].

\section{PH-targeted therapy}

$\mathrm{PH}$-targeted therapy is reserved for group 1 and $4 \mathrm{PH}$ patients only. Over the last years, treatment of SAPH using the PH-targeted therapy carefully shows some improvement in selected patients, especially in the presence of specific vasculopathy or increased vasoreactivity. This is based on case series only. Due to the lack of randomised controlled clinical trials and potential severe adverse events of treatment, the use of PH-targeted therapy in sarcoidosis is currently off-label. It should be noted that PH-targeted therapy can be ineffective and sometimes even dangerous for patients with fibrotic remodelling. These patients are less likely to respond to vasodilators, and treatment might worsen oxygenation, since the physiological hypoxaemic vasoconstriction is inhibited. As a result, there is an increased blood flow to areas with poor ventilation that might lead to a shunt or ventilation/perfusion discrepancy, as described in patients with idiopathic pulmonary fibrosis [43]. Therefore, significant hypoxia can be a contraindication for PH-targeted treatment. It may also cause acute pulmonary oedema and hypoxaemia through preferential vasodilation of the pulmonary arteries, if there is a down-stream venular obliteration by granulomas [16]. Therefore, treating physicians should be very careful before initiating these drugs in sarcoidosis patients, and treatment decisions should only be made on an individual basis in an expert centre by a multidisciplinary team, based on a goal-oriented approach with predefined targets.

Below, the PH-targeted therapies in SAPH will be discussed. Outcomes of the most important studies are summarised in Tab. 4.

\section{Prostacyclin analogues}

During the 1980s, prostanoids were the first agents used in an attempt to treat $\mathrm{PH}$ in sarcoidosis [44]. Decades later, a case series appeared evaluating epoprostenol for moderate to severe $\mathrm{PH}$ in eight sarcoidosis patients [16]. Most patients responded. One patient, with pulmonary veno-occlusive disease as an underlying mechanism of $\mathrm{PH}$, developed congestive heart failure leading to pulmonary oedema. In a study of 15 patients with inhaled iloprost [45], six had improved haemodynamics and significant improvement in quality of life. As a clinical outcome, only three patients had more than $30 \mathrm{~m}$ improvement in their 6MWT.

\section{Endothelin receptor antagonists}

Endothelin receptor antagonists have been more extensively investigated, particularly bosentan. One doubleblind, placebo-controlled trial investigated $35 \mathrm{PH}$ patients with all different sarcoidosis stages, presenting with New York Heart Association class II or III symptoms, low PAWP and stable on immunosuppressive therapy for at least three months [26]. Bosentan improved the mean PAP and pulmonary vascular resistance. However, this 16week period of treatment did not significantly improve the 6MWT or quality of life. A prospective case series of 21 sarcoidosis patients of all stages studied ambrisentan, another endothelin receptor antagonist. This resulted in preliminary cessation of treatment in $>50 \%$ of the patients [46], mainly due to oedema and/or dyspnoea. The ten patients who completed the study seemed to have an improved quality of life. However, the study was not powered to have significant results. There are no data regarding the outcomes of macitentan for treating $\mathrm{PH}$ in sarcoidosis. 


\section{Phosphodiesterase 5 inhibitors}

Milman and colleagues [9] reported improvement in the haemodynamics in patients with $\mathrm{PH}$ awaiting lung transplant, treated with sildenafil. There was no change in the 6MWT distance. Some cases suggest phosphodiesterase 5 inhibitors to be more successful in treating sarcoidosis patients with less severe $\mathrm{PH}[25,47]$.

\section{Lung transplant}

For patients with end-stage pulmonary parenchymal sarcoidosis or SAPH who failed therapy, single or bilateral lung transplant can be a feasible option. Between January 1995 and June 2012, 954 sarcoidosis patients out of 18 countries underwent lung transplant [48]. Median survival for sarcoidosis patients after lung transplant is calculated to be approximately 8.5 years [48].

\section{Conclusion}

Sarcoidosis patients have an increased risk of developing $\mathrm{PH}$. The exact prevalence of $\mathrm{PH}$ in sarcoidosis is unknown. To date, identifying sarcoidosis patients at risk for $\mathrm{PH}$ is still a major challenge. There is no validated algorithm to refer patients for $\mathrm{PH}$ analysis, which might lead to underdetection of PH. Moreover, echocardiography has limited diagnostic accuracy, especially in patients with parenchymal involvement. More extensive diagnostic tests and evaluation of treatment possibilities should therefore be considered in specialised centres with a multidisciplinary expert team. Treatment of $\mathrm{PH}$ in sarcoidosis is challenging, since there is a lack of robust data. Case series evaluating treatment options involving $\mathrm{PH}$-targeted therapy are arising for a subgroup of patients. Therefore, further research aiming to optimise the diagnostics and treatment for $\mathrm{PH}$ in pulmonary sarcoidosis is warranted.

Conflict of interest M.P. Huitema, J.C. Grutters, B.J.W.M. Rensing, H.J. Reesink and M.C. Post state that there are no conflicts of interest.

Open Access This article is distributed under the terms of the Creative Commons Attribution 4.0 International License (http:// creativecommons.org/licenses/by/4.0/), which permits unrestricted use, distribution, and reproduction in any medium, provided you give appropriate credit to the original author(s) and the source, provide a link to the Creative Commons license, and indicate if changes were made.

\section{References}

1. Iannuzzi MC, Rybicki BA, Teirstein AS. Sarcoidosis. N Engl J Med. 2007;357:2153-65.
2. Galiè N, Humbert M, Vachiery JL, et al. ESC/ERS Guidelines for the diagnosis and treatment of pulmonary hypertension. Eur Heart J. 2015;2015(37):67-119.

3. Bourbonnais JM, Samavati L. Clinical predictors of pulmonary hypertension in sarcoidosis. Eur Respir J. 2008;32:296-302.

4. Sulica R, Teirstein AS, Kakarla S, et al. Distinctive clinical, radiographic, and functional characteristics of patients with sarcoidosisrelated pulmonary hypertension. Chest. 2005;128:1483-89.

5. Handa T, Nagai S, Miki S, et al. Incidence of Pulmonary Hypertension and Its Clinical Relevance in Patients With Sarcoidosis. Chest. 2006;129:1246-52.

6. Alhamad E, Cal J, Alfaleh H, et al. Pulmonary hypertension in Saudi Arabia: A single center experience. Ann Thorac Med. 2013;8:78.

7. Baughman RP, Engel PJ, Taylor L, et al. Survival in sarcoidosisassociated pulmonary hypertension: The importance of hemodynamic evaluation. Chest. 2010;138:1078-85.

8. Shorr AF, Helman DL, Davies DB, et al. Pulmonary hypertension in advanced sarcoidosis: Epidemiology and clinical characteristics. Eur Respir J. 2005;25:783-88.

9. Milman N, Burton CM, Iversen M, et al. Pulmonary Hypertension in End-stage Pulmonary Sarcoidosis: Therapeutic Effect of Sildenafil? J Hear Lung Transpl. 2008;27:329-34.

10. Nunes H, Humbert M, Capron F, et al. Pulmonary hypertension associated with sarcoidosis: mechanisms, haemodynamics and prognosis. Thorax. 2006;61:68-74.

11. Shorr AF, Davies DB, Nathan SD. Predicting mortality in patients with sarcoidosis awaiting lung transplantation. Chest. 2003;124:922-28.

12. Smith LJ, Lawrence JB, Katzenstein AA. Vascular sarcoidosis: a rare cause of pulmonary hypertension. Am J Med Sci. 1983;285:38-44.

13. Takemura T, Matsui Y, Saiki S, et al. Pulmonary vascular involvement in sarcoidosis: A report of 40 autopsy cases. Hum Pathol. 1992;23:1216-23.

14. Rosen Y, Moon S, Huang C, et al. Granulomatous pulmonary angiitis in sarcoidosis. Arch Pathol Lab Med. 1977;101:170-74.

15. Preston IR, Klinger JR, Landzberg MJ, et al. Vasoresponsiveness of sarcoidosis-associated pulmonary hypertension. Chest. 2001;120:866-72.

16. Fisher KA, Serlin DM, Wilson KC, et al. Sarcoidosis-associated pulmonary hypertension: Outcome with long-term epoprostenol treatment. Chest. 2006;130:1481-88.

17. Damuth TE, Bower JS, Cho K, et al. Major pulmonary artery stenosis causing pulmonary hypertension in sarcoidosis. Chest. 1980;78:888-91.

18. Salazar A, Mañá J, Sala J, et al. Combined portal and pulmonary hypertension in sarcoidosis. Respiration. 1994;61:117-19.

19. Jones RM, Dawson A, Jenkins GH, et al. Sarcoidosis-related pulmonary veno-occlusive disease presenting with recurrent haemoptysis. Eur Respir J. 2009;34:517-20.

20. Joyce E, Ninaber MK, Katsanos S, et al. Subclinical left ventricular dysfunction by echocardiographic speckle-tracking strain analysis relates to outcome in sarcoidosis. Eur J Heart Fail. 2015;17:51-62.

21. Birnie DH, Sauer WH, Bogun F, et al. HRS expert consensus statement on the diagnosis and management of arrhythmias associated with cardiac sarcoidosis. Heart Rhythm. 2014;11:1305-23.

22. Maimon N, Salz L, Shershevsky Y, et al. Sarcoidosis-associated pulmonary hypertension in patients with near-normal lung function. Int J Tuberc Lung Dis. 2013;17:406-11.

23. Alhamad EH, Shaik SA, Idrees MM, et al. Outcome measures of the 6 minute walk test: relationships with physiologic and computed tomography findings in patients with sarcoidosis. BMC Pulm Med. 2010;10:42.

24. Keir GJ, Walsh SLF, Gatzoulis MA, et al. Treatment of sarcoidosisassociated pulmonary hypertension: A single centre retrospective 
experience using targeted therapies. Sarcoidosis Vasc Diffuse Lung Dis. 2014;31:82-90.

25. Barnett CF, Bonura EJ, Nathan SD, et al. Treatment of sarcoidosis-associated pulmonary hypertension: A two-center experience. Chest. 2009;135:1455-61.

26. Baughman RP, Culver A, Cordova F, et al. Bosentan for sarcoidosis-associated pulmonary hypertension: A double-blind placebo controlled randomized trial. Chest. 2014;145:810-17.

27. Baughman RP, Sparkman BK, Lower EE. Six-minute walk test and health status assessment in sarcoidosis. Chest. 2007;132:207-13.

28. Hoendermis ES. Pulmonary arterial hypertension: an update. Neth Heart J. 2011;19:514-22.

29. Armstrong HF, Schulze PC, Bacchetta M, et al. Impact of pulmonary hypertension on exercise performance in patients with interstitial lung disease undergoing evaluation for lung transplantation. Respirology. 2014;19:675-82.

30. Devaraj A, Wells AU, Meister MG, et al. The effect of diffuse pulmonary fibrosis on the reliability of CT signs of pulmonary hypertension. Radiology. 2008;249:1042-49.

31. Zisman DA, Karlamangla AS, Ross DJ, et al. High-resolution chest CT findings do not predict the presence of pulmonary hypertension in advanced idiopathic pulmonary fibrosis. Chest. 2007;132:773-79.

32. Huitema MP, Spee M, Vorselaars VMM, et al. Pulmonary artery diameter to predict pulmonary hypertension in pulmonary sarcoidosis. Eur Respir J. 2016;47:673-76.

33. Swift AJ, Rajaram S, Hurdman J, et al. Noninvasive estimation of PA pressure, flow, and resistance with CMR imaging: derivation and prospective validation study from the ASPIRE registry. JACC Cardiovasc Imaging. 2013;6:1036-47.

34. Ruocco G, Cekorja B, Rottoli P, et al. Role of BNP and echo measurement for pulmonary hypertension recognition in patients with interstitial lung disease: An algorithm application model. Respir Med. 2015;109:406-15.

35. Janda S, Shahidi N, Gin K, et al. Diagnostic accuracy of echocardiography for pulmonary hypertension: a systematic review and meta-analysis. Heart. 2011;97:612-22.

36. Arcasoy SM, Christie JD, Ferrari VA, et al. Echocardiographic assessment of pulmonary hypertension in patients with advanced lung disease. Am J Respir Crit Care Med. 2003;167:735-40.
37. Nathan SD, Shlobin OA, Barnett SD, et al. Right ventricular systolic pressure by echocardiography as a predictor of pulmonary hypertension in idiopathic pulmonary fibrosis. Respir Med. 2008;102:1305-10.

38. Patel MB, Mor-Avi V, Murtagh G, et al. Right Heart Involvement in Patients with Sarcoidosis. Echocardiography. 2016; [Epub ahead of print].

39. Hoeper MM, Lee SH, Voswinckel R, et al. Complications of right heart catheterization procedures in patients with pulmonary hypertension in experienced centers. J Am Coll Cardiol. 2006;48:254652.

40. Shorr F, Davies DB, Nathan SD. Outcomes for patients with sarcoidosis awaiting lung transplantation. Chest. 2002;122:233-38.

41. Seeger W, Adir Y, Barberà JA, et al. Pulmonary hypertension in chronic lung diseases. J Am Coll Cardiol. 2013;62(25 Suppl):D109-16.

42. Baughman RP, Grutters JC. New treatment strategies for pulmonary sarcoidosis: Antimetabolites, biological drugs, and other treatment approaches. Lancet Respir Med. 2015;3:813-22.

43. Pitsiou G, Papakosta D, Bouros D. Pulmonary hypertension in idiopathic pulmonary fibrosis: a review. Respiration. 2011;82:294-304.

44. Barst RJ, Ratner SJ. Sarcoidosis and reactive pulmonary hypertension. Arch Intern Med. 1985;145:2112-14.

45. Baughman RP, Judson MA, Lower EE, et al. Inhaled iloprost for sarcoidosis associated pulmonary hypertension. Sarcoidosis Vasc Diffus Lung Dis. 2009;26:110-20.

46. Judson MA, Highland KB, Kwon S, et al. Ambrisentan for sarcoidosis associated pulmonary hypertension. Sarcoidosis Vasc Diffus Lung Dis. 2011;28:139-45.

47. Dobarro D, Schreiber BE, Handler C, et al. Clinical characteristics, haemodynamics and treatment of pulmonary hypertension in sarcoidosis in a single centre, and meta-analysis of the published data. Am J Cardiol. 2013;111:278-85.

48. Yusen RD, Edwards LB, Kucheryavaya AY, et al. The registry of the international society for heart and lung transplantation: Thirtyfirst adult lung and heart-lung transplant report 2014; Focus theme: Retransplantation. J Heart Lung Transplant. 2014;33:1009-24. 